\section{MicroNova mit neuer Partner-Schnittstelle}

MicroNova, Anbieter von Vernetzungslösungen, hat eine Schnittstelle entwickelt, über die Hersteller von Praxisverwaltunssystemen (PVS) nun einen direkten Zugriff auf die Bausteine des MicroNova-Portfolios erlauben können. Module wie VisioDok oder das Abrechnungsmodul könnten in die PVS integriert werden. Bislang ermöglichte die Schnittstellentechnologie von MicroNova Praxen und weiteren Anwendern nur, vorhandene PVS direkt miteinander zu vernetzen. red.

\section{Digitale Gesundheitsangebote}

Laut einer Umfrage des Branchenverbands Bitkom unter gut 1.000 Bundesbürgern ist die Digitalisierung der Medizin durchaus im Trend. So würden $61 \%$ der Deutschen im Krankheitsfall einen OP-Roboter in Anspruch nehmen. Ein Viertel würde digitale Tabletten schlucken, ebenso viele würden die Informationen an ein Smartphone senden. Ein Drittel der Befragten ist außerdem offen für unter die Haut implantierbare Mikrochips zur Überwachung der Körperfunktionen. $15 \%$ würden diese sogar auf jeden Fall nutzen.

Rebekka Höhl

\section{Malware: Locky schlägt wieder zu}

Eine neue Version des VerschlüsselungsTrojaners Locky ist im Umlauf, so Bleepingcomputer.com. Diese versieht die verschlüsselten Dateien jetzt mit der Endung „..odin“. Bei den Vorgängerversionen wiesen „.locky" oder „.zepto" auf einen manifesten Schädlingsbefall hin. Eingeschleust wird der Trojaner nach wie vor über betrügerische E-Mails. Wer den Anhang öffnet, hat die Malware auf dem Rechner.

\section{Neuer Standard zur Risikoanalyse}

Hilfe bei der Analyse von Sicherheitslücken im Praxis-IT-System kann der neue Risikomanagement-Standard 200-3 des Bundesamts für Sicherheit in der Informationstechnik (BSI) bieten. Der Leitfaden ist etwas für den IT-Administrator oder technikaffinen Arzt. Erstmals werden in dem BSI-Standard alle risikobezogenen Arbeitsschritte bei der Umsetzung des IT-Grundschutzes gebündelt in einem Dokument dargestellt. Gleichzeitig wurde laut BSI das Gefährdungsmodell vereinfacht (tinyurl.com/zpj6zod). dikationsbasierte Medikationsmanagement (IMM), das derzeit die evidenz-basierte Verordnung von Fertigarzneimitteln und Wirkstoffen im Rahmen von Selektivverträgen abbildet. Viele der genannten Eigenschaften würden jetzt schon von IMM mitgeliefert. Und mit der Arzneimittelinitiative Sachsen-Thüringen (ARMIN), bei der die Wirkstoffverordnung und auch das gemeinsame Medikationsmanagement durch Arzt und Apotheker erprobt werden, leistet man technisch sogar schon mehr. Gehlen: „Wir sind an der Stelle schon recht weit."

\section{Warten auf die Richtlinie}

Das Problem liegt in den Vorgaben, die es für die Umsetzung der G-BA-Beschlüsse braucht. Hierfür müssen GKVSpitzenverband, KBV und eventuell auch die Bundesvereinigung Deutscher Apothekerverbände (ABDA) einen gemeinsamen Vorschlag erarbeiten, der zum einen in einer Richtlinie für die Ärzte, zum anderen aber in konkreten technischen Vorgaben für die Industrie münden müsste. Mit dem geplanten Inkrafttreten des AMNOG zum Jahresstart 2017 dürfte es damit noch keine passende EDV-Lösung geben, denn oft hängt es auch daran, dass sich die Vorgaben eben nicht an internationalen Standards der IT-Branche orientieren.

Proprietäre Ansätze bringen zum einen Widerstände aus den Softwarehäusern, zum anderen verlängern sie die Entwicklungszeit - und, es erhöhen sich die Softwarekosten für den Arzt. Würden sich die Krankenkassen tatsächlich an den Kosten beteiligen, hinterließe das wohl einen schalen Nachgeschmack: Zu groß wäre die Angst, dass damit die Therapiefreiheit des Arztes eingeschränkt wird und Kasseninteressen bei Verordnungen vorrangig bedient werden.

Die KBV hat aber noch einen anderen Plan: Sie würde die Arzneimitteldatenbank gerne generell unabhängig von der Praxissoftware sehen und am liebsten in die Telematikinfrastruktur integrieren. So könnten die Ärzte dann bei Bedarf schneller den Anbieter der Arzneidatenbank wechseln, heißt es. Das dürfte dahingehend ein Problem werden, dass die Telematikinfrastruk-

\section{Die AMNOG-Novelle}

Der vom Bundeskabinett am 12. Oktober 2016 verabschiedete Entwurf des "Gesetzes zur Stärkung der Arzneimittelversorgung in der GKV" - die AMNOG-Novelle - enthält unter anderem folgende Regelungen:

Ärzte sollen künftig über ihre Praxissoftware besser über die Ergebnisse der Nutzenbewertung informiert werden.

_ Die freie Preisbildung für ein Arzneimittel im ersten Jahr nach Markteinführung gilt künftig nur bis zum Erreichen eines Schwellenwertes in Höhe von 250 Millionen $€$.

_ Auch bei Zytostatika werden künftig Rabattverträge zwischen Krankenkassen und pharmazeutischen Herstellern erlaubt.

_ Das geltende Preismoratorium für Arzneimittel ohne Preisregulierung wird bis zum Ende des Jahres 2022 verlängert.

- Die Besonderheiten von Kinderarzneimitteln sollen bei der Nutzenbewertung noch besser berücksichtigt werden können.

tur de facto bislang nicht existiert und sich die ersten Online-Tests wohl in das nächste Frühjahr verschieben - und nur mit etwas Glück in der Region Nordwest vielleicht noch in diesem Jahr anlaufen. Bei allen bisherigen Erfahrungen mit der Telematikinfrastruktur dürfte auch die Arzneimitteldatenbank selbst dann wohl kaum zeitnah zur Verfügung stehen.

Von einer Arzneimitteldatenbank in der Cloud hält zumindest Gehlen nichts: „Was passiert, wenn der Zugriff während der Praxiszeiten nicht möglich ist?", fragt er. Und es müsse sichergestellt werden, dass die Cloud-Lösung dem Leistungsanspruch der Praxen gerecht wird. „Das Rezeptformular wird in großen Praxen mehrere hundert Mal an einem Morgen befüllt. Jede Verzögerung durch langsame Zugriffe auf die Medikamentendatenbank sind ein No-Go." Zum anderen sei in vielen ländlichen Regionen noch kein schneller Internetzugang verfügbar.

RebekkaHöhl 